\title{
Injury of the ileum during percutaneous nephrolithotomy in a pediatric patient
}

\author{
Karim S. M. Saad, MD; ${ }^{*}$ Ahmed Hanno, MD; ${ }^{*}$ Ahmed R. El-Nahas, MD ${ }^{\dagger}$ \\ "Urology Department, Faculty of Medicine, Alexandria University, Alexandria, Egypt; 'Urology Department, Urology and Nephrology Center, Mansoura University, Mansoura, Egypt
}

Cite as: Can Urol Assoc J 2014;8(3-4):e204-6. http://dx.doi.org/10.5489/cuaj.1644 Published online March 11, 2014.

\section{Abstract}

Ileum injury during percutaneous nephrolithotomy (PCNL) is an extremely rare complication. We describe the successful management of an inadvertently injured ileum during subcostal PCNL in a 12-year-old boy. Mechanism of injury, presentation and management will be discussed.

\section{Introduction}

Percutaneous nephrolithotomy (PCNL) is a well-established treatment for complex or multiple renal stones. It is a safe procedure, but associated with specific complications. ${ }^{1}$ Most are low-grade complications, such as postoperative fever or urinary leakage. Major complications, such as renal bleeding necessitating blood transfusion and pleura injury, are rare, ${ }^{2}$ while injuries to adjacent organs, such as the colon or liver, are very rare. ${ }^{3,4}$ For the small bowel, only 1 case of duodenal injury has been reported. ${ }^{5}$ In pediatric patients, a case report of colonic injury during PCNL has been recently published. ${ }^{6}$

This case report describes an extremely rare complication. To the best of our knowledge, this is the first case reports of ileum injury during PCNL in a pediatric patient.

\section{Case report}

A 12-year-old boy presented with recurrent right flank pain for 4 months. Plain x-ray film and intravenous urography showed multiple right radio-opaque renal stones with hydronephrosis (Fig. 1a, Fig. 1b). The child's body mass index was 24.3. The PCNL was performed while the patient was in the prone position. A 5-French (Fr) ureteral catheter was placed through the ureteral orifice and iodinated contrast was installed to opacified the renal pelvis and calyces. Subcostal puncture targeting the posterior lower calyx was established by an endourologist under fluoroscopic guidance using a multidirectional C-arm. The skin puncture was medial to the posterior axillary line. The tract was dilated using Alken's coaxial dilators, and an Amplatz sheath of 18-Fr was fixed. A 15-Fr Mini-nephroscope (Karl Storz Endoskope, Tuttlingen, Germany) was used to retrieve all stones after disintegration with pneumatic lithotrite. After removing the impacted stone, we confirmed the patency of the ureteropelvic junction with antegrade nephrostogram. The operation was completed without intraoperative complications. At the end of the procedure, the kidney was drained with a ureteral catheter without nephrostomy tube (tubeless PCNL).

On the first postoperative day, the patient developed abdominal pain and fever. His abdomen was distended and tender with sluggish intestinal sounds. Ultrasound revealed free intraperitoneal fluid. Computed tomography (CT) confirmed the presence of intraperitoneal fluid and showed the inlet of the nephrostomy tract (Fig. 2). Colonic injury was suspected, and urgent open exploration was performed by a pediatric surgeon.

Midline incision was used for exploration and the peritoneal cavity was cleared from the intestinal contents then irrigated with warm saline. The ascending colon was completely free on thorough examination. Examination of abdominal organs revealed a transfixing ileal injury with inlet and outlet at $20 \mathrm{~cm}$ from the ileo-caecal junction (Fig. 3). Suturing of injured ileum was done with polyglactine 4/0 sutures and an intraperitoneal tube drain was left. The postoperative period was uneventful and oral feeding resumed after 5 days at which time the patient was discharged.

\section{Discussion}

Bowel injury during PCNL is a very rare complication. There are two mechanisms that explain this injury. The first is the retrorenal position of the intestinal segment that leads to injury of the bowel before percutaneous access reaches the kidney. Therefore, an inlet and outlet injuries of the bowel are identified. This mechanism was more noticed with colonic injury because retrorenal position of the colon was identified in $15 \%$ on multiplaner reformatted CT. ${ }^{7}$ The 


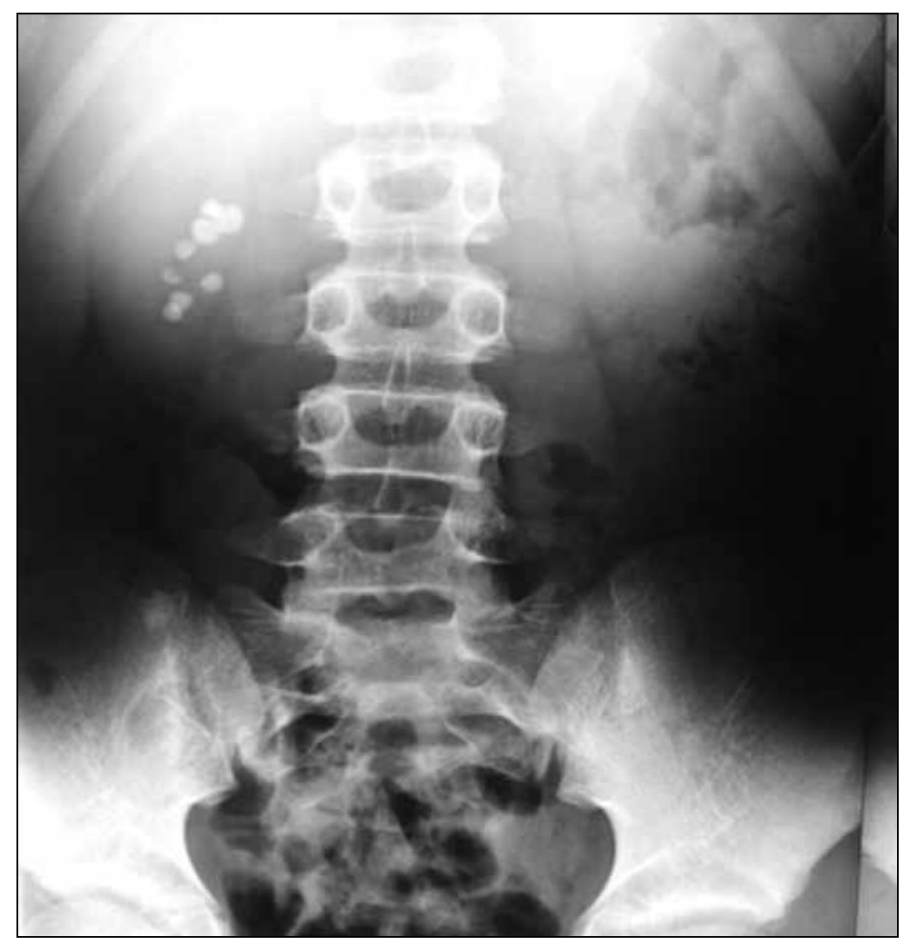

Fig. 1a. A plain X-ray film of the abdomen showing multiple radio-opaque shadows in the right kidney region.

second mechanism explains duodenal injury when the renal pelvis is inadvertently perforated during the right PCNL because the duodenum is present antero-medially to the renal pelvis. ${ }^{5}$ Therefore, an inlet injury of the duodenum will be seen. In our case, the first mechanism was the most probable explanation because there were an inlet and outlet

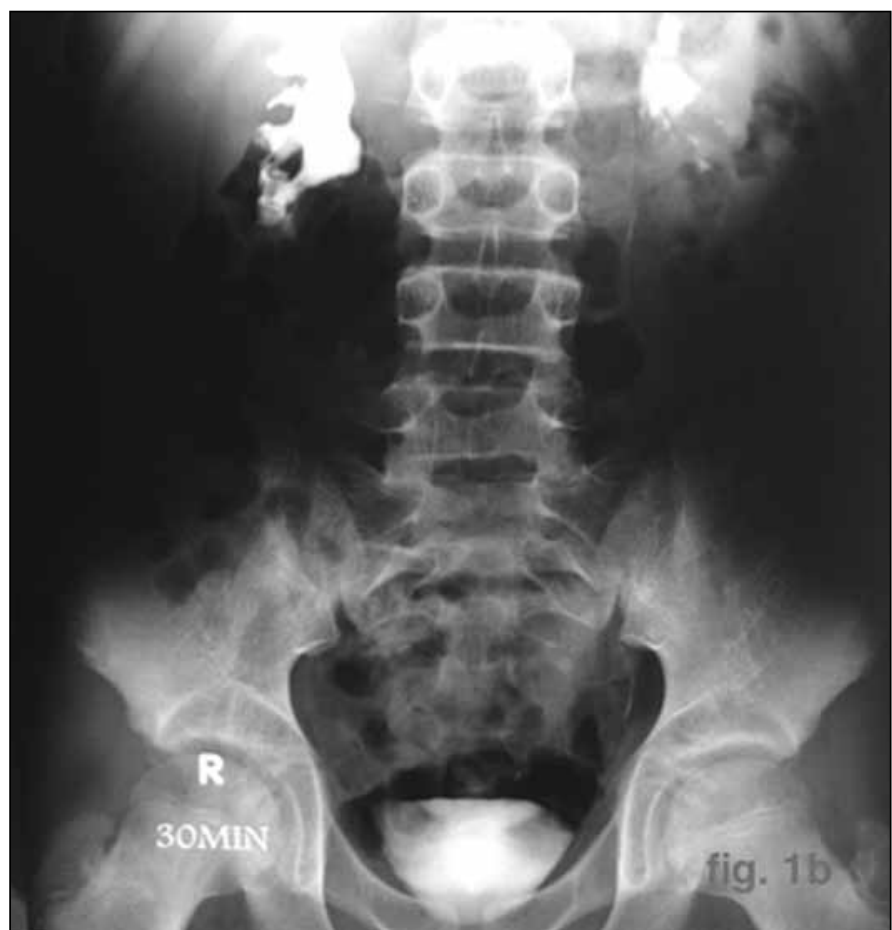

Fig. $\mathbf{1 b}$. Intravenous urography showing right hydronephrosis.

injuries of the ileum, and no perforation of the renal pelvis during PCNL. It is very rare to find the ileum posterior to the kidney. However, the incidence of retrorenal colon occurs more in thin patients, where the perinephric fat is minimal and the space behind the kidney is empty. ${ }^{8}$ In our case, the ileal segment was in a peritoneal recess behind the lower pole of kidney at time of surgery.

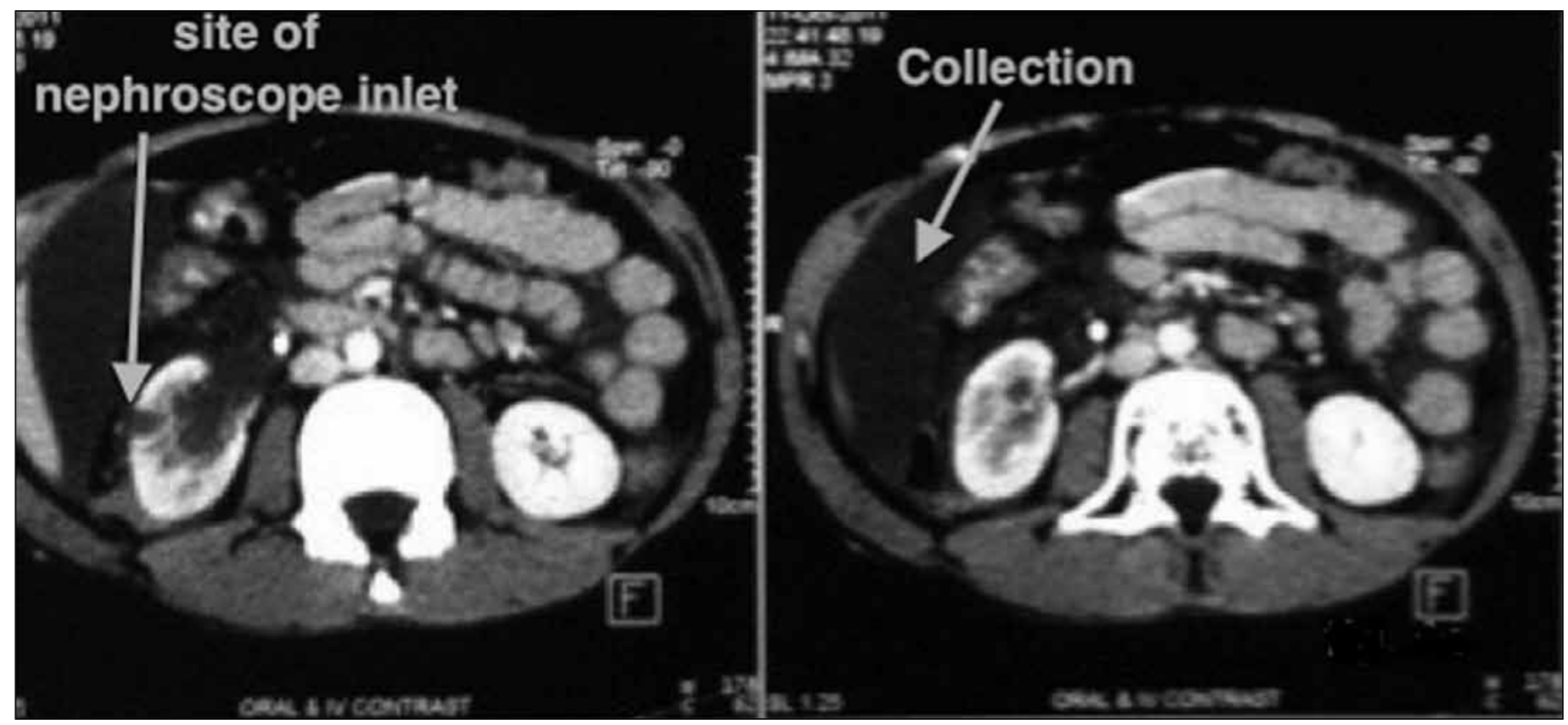

Fig. 2. Non-contrast computed tomography, axial scans showing intrapeitoneal fluid and the site of the nephrostomy inlet in the renal parenchyma. 


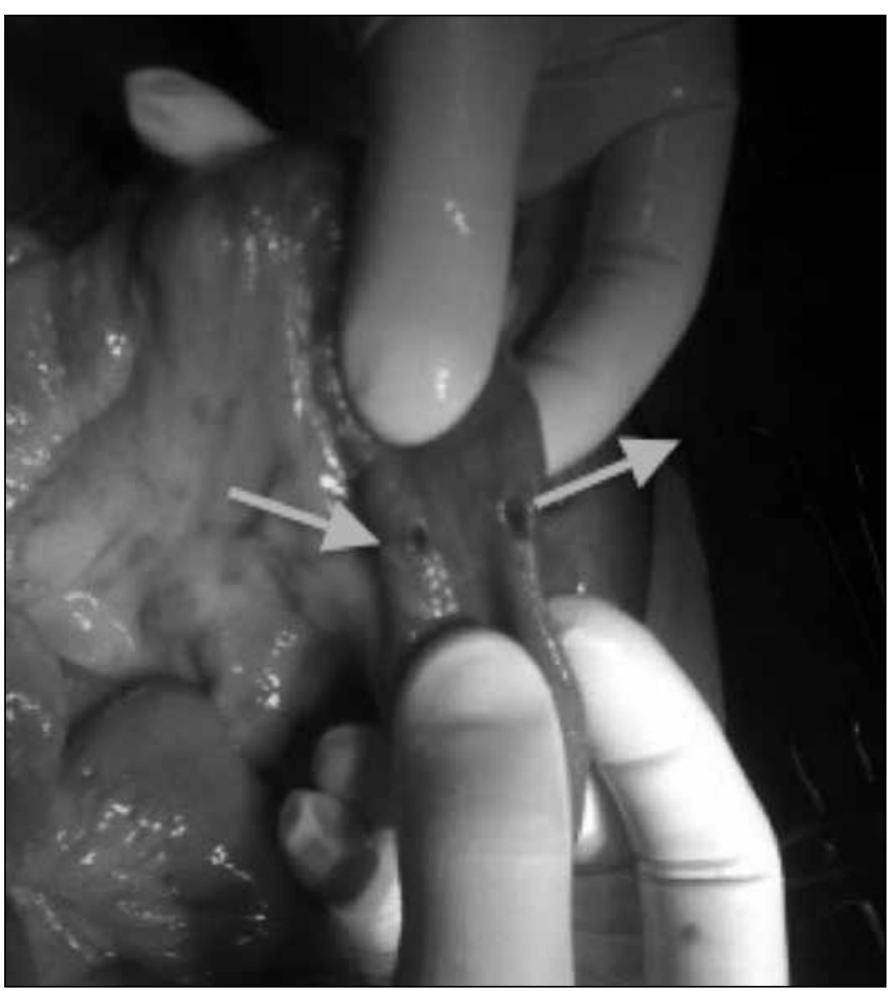

Fig. 3. Intraoperative photography showing the inlet and exit injuries of the ileum.

In our patient, the presentation was abdominal pain, distention and fever; the peritoneum was contaminated by the intestinal contents as no nephrostomy tube was placed. This is different from the case report of Winer and colleagues; their patient developed small bowel obstruction due to the presence of a nephrostomy tube. ${ }^{9}$ However, the injury may be diagnosed early if an antegrade nephrostogram through a nephrostomy tube is performed.

The present complication could not have been avoided because of the retrorenal ileum in patients without renal anomalies. Intraoperative techniques to minimize or even avoid intestinal injury during PCNL include: ultrasound guided puncture ${ }^{10}$ and observation of the intestinal gas shadow posterior to kidney in the lateral view of the fluoroscopic-guided puncture. ${ }^{3}$

Conservative treatment of retroperitoneal bowel injury (either colonic ${ }^{3}$ or duodenal ${ }^{5}$ ) is usually successful. Exploration was needed in cases of intraperitoneal injuries that lead to contamination of the peritoneal cavity with intestinal contents resulting in fever, sepsis or peritonitis. In the present case, we decided to explore when fluid was seen inside the peritoneal cavity on CT scans. Simple sutures of the inlet and outlet injuries of the ileum without proximal intestinal diversion were successful because there was no thermal injury and the ileal wall was healthy. However, if there is any doubt about the vitality of the injured ileal segment, resection of the injured area with intestinal reanastmosis is advised.

\section{Conclusion}

To the best of our knowledge, this is the first case report of ileum injury during PCNL in pediatric patients. Injury of the ileum during PCNL is an extremely rare complication because the ileum is present anterior to the kidney inside the peritoneal cavity, whereas the percutaneous access is retroperitoneal through the posterior surface of the kidney. Does the peritoneal relation vary in children compared to adults? The differences in the relation between the kidney and ileum in children have not yet been studied. We hope this case report encourages further studies, such as CT scans in supine and prone positions.

Competing interests: Dr. Saad, Dr. Hanno and Dr. El-Nahas all declare no competing financial or personal interests.

This paper has been peer-reviewed.

\section{References}

1. Michel MS, Trojan L, Rassweiler JJ. Complications in percutaneous nephrolithotomy. Eur Urol 2007;51:899906. http://dx.doi.org/10.1016/i.eururo.2006.10.020

2. Tefekli A, Ali Karadag M, Tepeler K, et al. Classification of percutaneous nephrolithotomy complications using the modified Clavien grading system: Looking for a standard. Eur Urol 2008;53:184-90. http://dx.doi.org/10.1016/i.eururo.2007.06.049

3. El-Nahas AR, Shokeir AA, El-Assmy AM, et al. Colonic perforation during percutaneous nephrolithotomy: Study of risk factors. Urology 2006;67:837-941. http://dx.doi.org/10.1016/i.urology.2005.11.025

4. El-Nahas AR, Mansour AM, Ellaithy R, et al. Conservative treatment of liver injury during percutaneous nephrolithotomy. J Endourol 2008;22:1649-52. http://dx.doi.org/10.1089/end.2008.0147

5. Culkin DJ, Wheeler JS Jr, Canning JR. Nephro-duodenal fistula: A complication of percutaneous nephrolithotomy. J Urol 1985;134:528-30.

6. Goger $E$, Guven $S$, Gurbuz $R$, et al. Management of a colon perforation during pediatric percutaneous nephrolithotomy. J Endourol 2012;26:1118-20. http://dx.doi.org/10.1089/end.2011.0433

7. Tuttle DN, Yeh BM, Meng MV, et al. Risk of injury to adjacent organs with lower-pole fluoroscopically guided percutaneous nephrostomy: Evaluation with prone, supine, and multiplanar reformatted CT. J Vasc Interv Radiol 2005;16:1489-92. http://dx.doi.org/10.1097/01.RVI.0000175331.93499.44

8. Traxer 0 . Management of injury to the bowel during percutaneous stone removal. J Endourol 2009;23:1777-80. http://dx.doi.org/10.1089/end.2009.1553

9. Winer $A G$, Hyams ES, Shah 0 . Small bowel injury during percutaneous nephrostomy tube placement causing small bowel obstruction. Can I Urol 2009;16:4950-2.

10. Osman $M$, Wendt-Nordahl $G$, Heger $K$, et al. Percutaneous nephrolithotomy with ultrasonography-guided renal access: Experience from over 300 cases. BJU Int 2005;96:875-8. http://dx.doi.org/10.1111/i.1464-410X.2005.05749.x

Correspondence: Dr. Ahmed R. El-Nahas, Assistant Professor of Urology, Urology and Nephrology Center, Mansoura University, Mansoura, Egypt; ar_el_nahas@yahoo.com 